\title{
Rotational Saturation Properties of Isotropic Vector Hysteresis Models Using Vectorized Stop and Play Hysterons
}

AUTHOR(S):

Matsuo, Tetsuji

\section{CITATION:}

Matsuo, Tetsuji. Rotational Saturation Properties of Isotropic Vector Hysteresis Models Using Vectorized Stop and Play Hysterons. IEEE TRANSACTIONS ON MAGNETICS 2008, 44(11(Part 1)): 3185-3188

\section{ISSUE DATE:}

2008-11

URL:

http://hdl.handle.net/2433/84540

\section{RIGHT:}

(c) 2008 IEEE. Personal use of this material is permitted. However, permission to reprint/republish this material for advertising or promotional purposes or for creating new collective works for resale or redistribution to servers or lists, or to reuse any copyrighted component of this work in other works must be obtained from the IEEE. 


\title{
Rotational Saturation Properties of Isotropic Vector Hysteresis Models Using Vectorized Stop and Play Hysterons
}

\author{
Tetsuji Matsuo \\ Department of Electrical Engineering, Kyoto University, Kyoto 615-8510, Japan
}

\begin{abstract}
This article discusses saturation properties of two-dimensional isotropic vector hysteresis models using geometrically vectorized stop and play hysterons. The vector stop and play hysterons are modified to obtain natural saturation properties. The modified hysterons improve rotational properties of vector stop and play models under a saturated condition.
\end{abstract}

Index Terms-Play hysteron, rotational hysteresis loss, saturation, stop hysteron, vector hysteresis.

\section{INTRODUCTION}

STOP and play models [1], [2] are efficient and precise hysteresis models that can accurately represent the scalar hysteretic properties of silicon steel sheets [3]-[5].

Two types of vectorized play models have been proposed: one is a superposition of scalar models [6], whereas the other is a geometrical vectorization of a play hysteron [1], [6]-[8]. Both vector play models have proven able to adjust their rotational hysteresis losses to a measured loss [6]. The latter model is, however, more efficient than the former, which requires integration along the azimuthal direction.

Similarly to the vectorized play hysteron, a stop hysteron is also geometrically vectorized. The hysteresis model using the vectorized stop hysteron [9] can be an efficient model that directly provides an output vector of the magnetic field from an input vector of magnetic flux density. It is computationally less expensive than the vector stop model that is a superposition of scalar models [10], [11]. However, properties of the hysteresis model using the vectorized stop hysteron have not yet been sufficiently discussed.

This paper discusses the saturation property of the vectorized stop hysteron. The vector stop hysteron is modified to obtain natural rotational properties. The modification is applied also to the vector play hysteron. The rotational properties of two-dimensional hysteresis models are then examined using the vector stop and play hysterons comparing with a measured property of a silicon steel sheet.

\section{Vector Play AND StoP Hysterons}

A vectorized play hysteron [7] $\boldsymbol{p}_{\eta}$ is given as

$$
\boldsymbol{p}_{\eta}(\boldsymbol{B})=\boldsymbol{B}-\frac{\eta\left(\boldsymbol{B}-\boldsymbol{p}_{\eta}^{0}\right)}{\max \left(\eta,\left|\boldsymbol{B}-\boldsymbol{p}_{\eta}^{0}\right|\right)}
$$

where $\eta$ is a hysteron radius and $\boldsymbol{p}_{\eta}^{0}$ is the vector $\boldsymbol{p}_{\eta}$ at the previous time-point. The geometrical meaning of the vector play hysteron is illustrated in Fig. 1. Point $\mathrm{B}(\overrightarrow{\mathrm{OB}}=\boldsymbol{B})$ is located in a circle (or sphere for a 3-D hysteron) for which the center and radius are $\mathrm{P}\left(\overrightarrow{\mathrm{OP}}=p_{\eta}\right)$ and $\eta$, respectively. When $\mathrm{B}$ moves
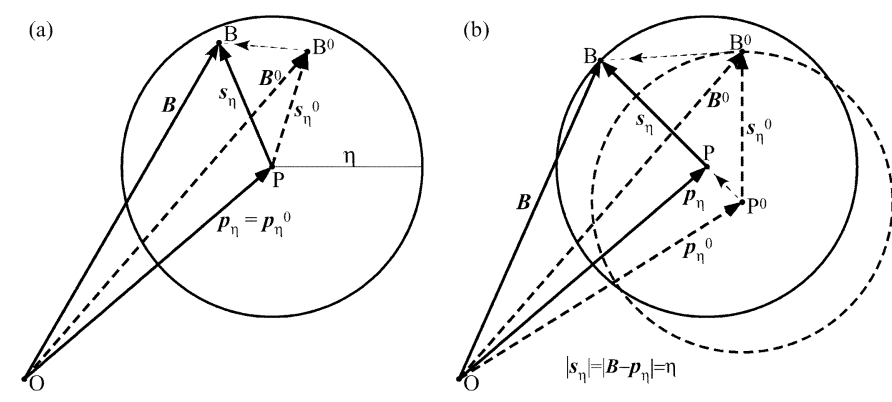

Fig. 1. Vector play and stop hysterons. (a) $\left|\boldsymbol{B}-\boldsymbol{p}_{\eta}^{0}\right| \leq \eta$. (b) $\left|\boldsymbol{B}-\boldsymbol{p}_{\eta}^{0}\right|>\eta$.

inside the circle, P does not move [Fig. 1(a)]. When B reaches the circumference of the circle, the circle and its center $\mathrm{P}$ move together with B [Fig. 1(b)].

A stop hysteron $\boldsymbol{s}_{\eta}(\boldsymbol{B})$ is defined complementarily to a play hysteron [1] as

$$
\boldsymbol{s}_{\eta}(\boldsymbol{B})=\boldsymbol{B}-\boldsymbol{p}_{\eta}(\boldsymbol{B}) .
$$

The vector play hysteron (1) is accordingly accompanied by the stop hysteron given as

$$
\begin{aligned}
\boldsymbol{s}_{\eta}(\boldsymbol{B}) & =\frac{\eta\left(\boldsymbol{B}-\boldsymbol{p}_{\eta}^{0}\right)}{\max \left(\eta,\left|\boldsymbol{B}-\boldsymbol{p}_{\eta}^{0}\right|\right)} \\
& =\frac{\eta\left(\boldsymbol{B}-\boldsymbol{B}^{0}+\boldsymbol{s}_{\eta}^{0}\right)}{\max \left(\eta,\left|\boldsymbol{B}-\boldsymbol{B}^{0}+\boldsymbol{s}_{\eta}^{0}\right|\right)}
\end{aligned}
$$

where $\boldsymbol{B}^{0}$ and $\boldsymbol{s}_{\eta}^{0}=\boldsymbol{B}^{0}-\boldsymbol{p}_{\eta}^{0}$ are the vectors $\boldsymbol{B}$ and $\boldsymbol{s}_{\eta}$ at the previous time-point.

When a rotational input

$$
\boldsymbol{B}=\left(B_{\mathrm{a}} \cos \omega t, B_{\mathrm{a}} \sin \omega t\right)
$$

is given, $\boldsymbol{s}_{\eta}$ becomes perpendicular to $\boldsymbol{p}_{\eta}$ at steady state as shown in Fig. 2(a) when $B_{\mathrm{a}}>\eta$. When $B_{\mathrm{a}} \leq \eta, \boldsymbol{p}_{\eta}$ becomes a constant vector $\boldsymbol{p}_{\eta}^{\mathrm{C}}\left(\left|\boldsymbol{p}_{\eta}^{\mathrm{C}}\right| \leq \eta-B_{\mathrm{a}}\right)$ and $\boldsymbol{s}_{\eta}$ is given by $\boldsymbol{B}-\boldsymbol{p}_{\eta}^{\mathrm{C}}$ for the input (4).

\section{VECTOR STOP MODEL}

Digital Object Identifier 10.1109/TMAG.2008.2001656

Color versions of one or more of the figures in this paper are available online at http://ieeexplore.org.

\section{A. Hysteresis Model Using a Vectorized Stop Hysteron}

As illustrated in Fig. 2(a), the stop hysteron $\boldsymbol{s}_{\eta}$ usually has a phase lead to the input $\boldsymbol{B}$, whereas $\boldsymbol{p}_{\eta}$ has a phase lag to $\boldsymbol{B}$. 
(a)

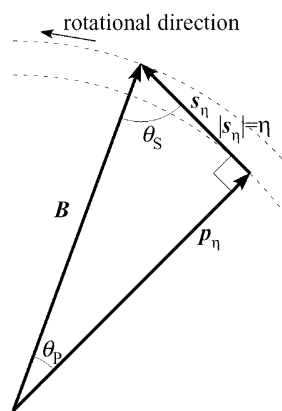

(b)

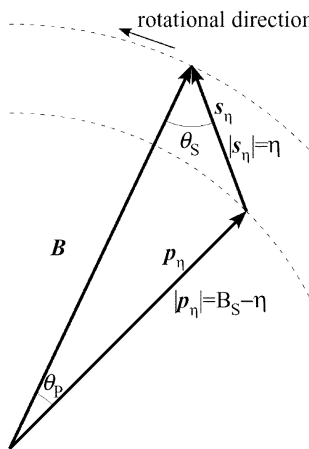

(c)

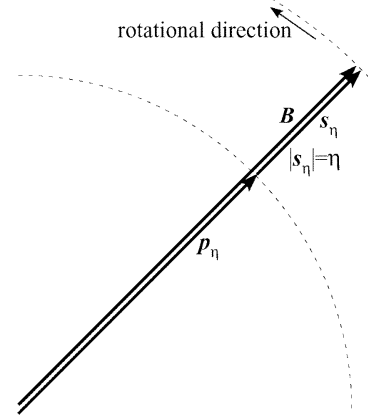

Fig. 2. Vector relations for rotational inputs of $\boldsymbol{B}$.

Accordingly, it is natural for the stop model to represent a hysteretic relation from an input vector of magnetic flux density $\boldsymbol{B}$ to an output vector of magnetic field $\boldsymbol{H}$.

Using the stop hysteron (3), a vector stop model is given as

$$
\begin{aligned}
\boldsymbol{H} & =\boldsymbol{S}(\boldsymbol{B})=\int_{0}^{B_{\mathrm{S}}} \boldsymbol{g}\left(\eta, \boldsymbol{s}_{\eta}(\boldsymbol{B})\right) \mathrm{d} \eta \\
\boldsymbol{g}(\eta, \boldsymbol{s}) & =g(\eta,|\boldsymbol{s}|) \frac{\boldsymbol{s}}{|\boldsymbol{s}|}
\end{aligned}
$$

where $g(\eta, s)$ is a scalar shape function and $B_{\mathrm{S}}$ is the saturation magnetic flux density. An anisotropic version of the vector stop model has been discussed in [9].

The vector model (5) using (3) exhibits a hysteretic property even when $|\boldsymbol{B}|>B_{\mathrm{S}}$. A scalar stop model discussed in [3] uses a redefined stop hysteron to suppress the hysteretic property after saturation. Its natural extension to a vector version is given as

$$
\boldsymbol{s}_{\eta}(\boldsymbol{B})=\frac{\eta\left(\boldsymbol{B}-\boldsymbol{B}^{0 *}+\boldsymbol{s}_{\eta}^{0}\right)}{\max \left(\eta,\left|\boldsymbol{B}-\boldsymbol{B}^{0 *}+\boldsymbol{s}_{\eta}^{0}\right|\right)}
$$

where

$$
\boldsymbol{B}^{0 *}=\frac{B_{\mathrm{S}} \boldsymbol{B}^{0}}{\max \left(B_{\mathrm{S}},\left|\boldsymbol{B}^{0}\right|\right)} .
$$

For the rotational input (4), $\boldsymbol{s}_{\eta}$ given by (7) and (8) becomes parallel to $B$ at steady state as shown in Fig. 2(c) for $B_{\mathrm{a}}>B_{\mathrm{S}}$.

Another way to prohibit the hysteretic property after the saturation is given as

$$
\boldsymbol{s}_{\eta}(\boldsymbol{B})=\frac{\eta\left(\boldsymbol{B}-\boldsymbol{p}_{\eta}^{0 *}\right)}{\max \left(\eta,\left|\boldsymbol{B}-\boldsymbol{p}_{\eta}^{0 *}\right|\right)}
$$

where

$$
\begin{aligned}
\boldsymbol{p}_{\eta}^{0 *} & =\frac{\left(B_{\mathrm{S}}-\eta\right) \boldsymbol{p}_{\eta}^{0}}{\max \left(B_{\mathrm{S}}-\eta,\left|\boldsymbol{p}_{\eta}^{0}\right|\right)} \\
& =\frac{\left(B_{\mathrm{S}}-\eta\right)\left(\boldsymbol{B}^{0}-\boldsymbol{s}_{\eta}^{0}\right)}{\max \left(B_{\mathrm{S}}-\eta,\left|\boldsymbol{B}^{0}-\boldsymbol{s}_{\eta}^{0}\right|\right)}
\end{aligned}
$$

The stop hysteron (9) is equivalent to (7) for unidirectional inputs. The response of (9) differs from those of (3) and (7) for the rotational input (4) when $B_{\mathrm{T}}<B_{\mathrm{a}}<B_{\mathrm{S}}$, where $B_{\mathrm{T}}=$ $\sqrt{\left(B_{\mathrm{S}}-\eta\right)^{2}+\eta^{2}}$. When $B_{\mathrm{T}}<B_{\mathrm{a}}<B_{\mathrm{S}},\left|\boldsymbol{B}-\boldsymbol{s}_{\eta}\right|$ becomes $B_{\mathrm{S}}-\eta$ at steady state as shown in Fig. 2(b).
TABLE I

VECTOR RELATIONS FOR THE ROTATIONAL INPUT OF $\boldsymbol{B}$

\begin{tabular}{cc|ccc|}
\hline Type & Eqs. & $\eta<|\boldsymbol{B}|<B_{\mathrm{T}}$ & $B_{\mathrm{T}}<|\boldsymbol{B}|<B_{\mathrm{S}}$ & $B_{\mathrm{S}}<|\boldsymbol{B}|$ \\
\hline (o) & (3) & Fig. 2 (a) & Fig. 2 (a) & Fig. 2 (a) \\
(s) & (7) and (8) & Fig. 2 (a) & Fig. 2 (a) & Fig. 2 (c) \\
(p) & (9) and (10) & Fig. 2 (a) & Fig. 2 (b) & Fig. 2 (c) \\
\hline
\end{tabular}

Vector relations for steady-state rotational inputs are summarized in Table I and Fig. 2, where three types of stop hysterons are compared: hysteron (o) — the original type using (1); hysteron (s) - the modified type using (7) and (8); and hysteron (p) - the modified type using (9) and (10).

As shown in Fig. 2, the angle between $\boldsymbol{B}$ and $\boldsymbol{s}_{\eta}$ is denoted by $\theta_{\mathrm{S}}$. The stop hysteron (o) gives $\theta_{\mathrm{S}} \rightarrow \pi / 2$ for $B_{\mathrm{a}} \rightarrow \infty$. When the stop hysteron (s) is used, $\theta_{S}$ discontinuously changes from $\cos ^{-1}\left(\eta / B_{\mathrm{a}}\right)$ [Fig. 2(a)] to zero [Fig. 2(c)] at $B_{\mathrm{a}}=B_{\mathrm{S}}$. The stop hysteron (p) achieves a continuous angle change, which naturally corresponds to the fact that $\boldsymbol{B}$ becomes parallel to $\boldsymbol{H}$ for a large rotational magnetic field.

\section{B. Hysteresis Losses}

The vector hysteresis model (5) using stop hysterons (o), (s), and (p) is referred to as vector stop models (o), (s), and (p), respectively, hereafter.

For an alternating input with amplitude $B_{\mathrm{a}}$, the vector stop models yield rate-independent hysteresis losses per cycle as

$L_{\mathrm{alt}}\left(B_{\mathrm{a}}\right)=\left\{\begin{array}{l}\left.\int_{0}^{B_{\mathrm{m}}} 4\left(B_{\mathrm{a}}-\eta\right) g(\eta, \eta) \mathrm{d} \eta \text { (model }(\mathrm{o})\right) \\ \left.\int_{0}^{B_{\mathrm{m}}} 4\left(B_{\mathrm{m}}-\eta\right) g(\eta, \eta) \mathrm{d} \eta \text { (models }(\mathrm{s}) \text { and }(\mathrm{p})\right)\end{array}\right.$

where $g(\eta,-B)=-g(\eta, B)$ is assumed and

$$
B_{\mathrm{m}}=\min \left(B_{\mathrm{a}}, B_{\mathrm{S}}\right) \text {. }
$$

The integrands in (11) mean the area of $B-g$ loop [11]. The alternating hysteresis loss of stop models (s) and (p) saturates when $B_{\mathrm{a}} \geq B_{\mathrm{S}}$, whereas that of (o) increases with $B_{\mathrm{a}}$ even when $B_{\mathrm{a}} \geq B_{\mathrm{S}}$.

For the rotational input (4), the vector stop models yield rateindependent hysteresis losses per cycle of

$$
L_{\mathrm{rot}}\left(B_{\mathrm{a}}\right)=2 \pi \int_{0}^{B_{\mathrm{m}}} g(\eta, \eta) B_{\mathrm{a}} \sin \theta_{\mathrm{S}} \mathrm{d} \eta .
$$



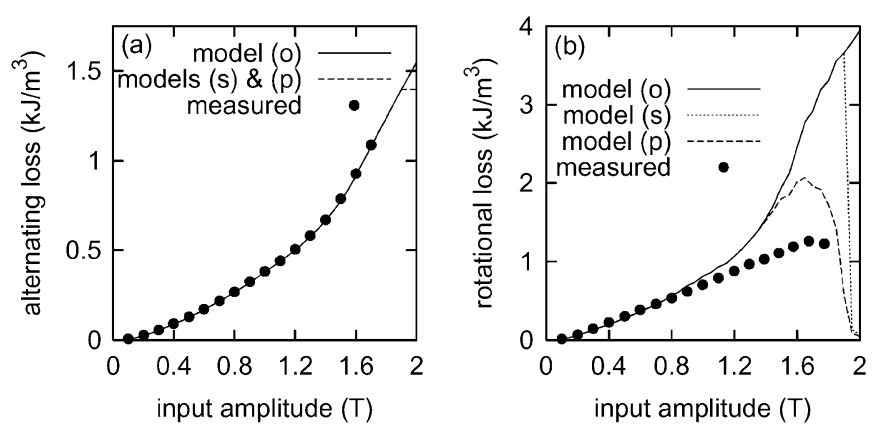

Fig. 3. Hysteresis losses per cycle simulated by vector stop models. (a) Alternating loss. (b) Rotational loss.

The vector relation shown in Fig. 2(a) gives $B_{\mathrm{a}} \sin \theta_{\mathrm{S}}=$ $\sqrt{B_{\mathrm{a}}{ }^{2}-\eta^{2}}$, whereas it becomes zero for the relation in Fig. 2(c). The vector relation shown in Fig. 2(b) gives $\theta_{\mathrm{S}}$ as

$$
\theta_{\mathrm{S}}=\cos ^{-1} \frac{B_{\mathrm{a}}{ }^{2}+\eta^{2}-\left(B_{\mathrm{S}}-\eta\right)^{2}}{2 B_{\mathrm{a}} \eta} .
$$

\section{Simulation of Vector Property}

Hysteretic properties of a non-oriented silicon steel sheet (JIS: 50A1300) were measured using a rotational single sheet tester. Its anisotropic property is averaged along the azimuthal direction to obtain an isotropic property for simulations.

For unidirectional inputs, the vector model (5) is reduced to a scalar model having the same shape function $g(\eta, s)$. This means that $g(\eta, s)$ can be determined only from unidirectionally measured data. The stop model is discretized with 38 hysterons and their shape functions are identified using the method described in [10]. $B_{\mathrm{S}}$ is set to $1.9 \mathrm{~T}$ because of the lack of measured data for large $B$ although $1.9 \mathrm{~T}$ is small compared with the saturation magnetization of steel sheet.

Fig. 3 shows the simulated and measured hysteresis losses. Model (o) has monotonically increasing alternating and rotational losses. Model (s) has a sharp decrease in the rotational loss at $B_{\mathrm{S}}$. Model (p) has an acceptably natural loss property in that the rotational loss continuously decreases for large $B_{\mathrm{a}}$. The discrepancy with the measured loss can be reduced using a weighting function as shown for the vector play model in [6]. The rotational losses given by models (s) and (p) become zero when $B_{\mathrm{a}} \geq B_{\mathrm{S}}$ because $\boldsymbol{s}_{\eta}$ becomes parallel to $\boldsymbol{B}$, while the alternating losses saturate when $B_{\mathrm{a}} \geq B_{\mathrm{S}}$. However, $B_{\mathrm{S}}$ used in the simulation is too small for the rotational loss to become zero and for the alternating loss to saturate. Measured data for large $\boldsymbol{B}$ are required to discuss saturation properties quantitatively.

\section{Vector Play Model}

\section{A. Hysteresis Model Using a Vectorized Play Hysteron}

The play hysteron usually has a phase lag to its input. However, the vector play model can be used to represent a hysteretic relation from an input of magnetic flux density $\boldsymbol{B}$ to an output of magnetic field $\boldsymbol{H}$ [6].

The vector model using (1) is given similarly to (5) as

$$
\begin{gathered}
\boldsymbol{H}=\boldsymbol{P}(\boldsymbol{B})=\int_{0}^{B_{\mathrm{S}}} \boldsymbol{f}\left(\eta, \boldsymbol{p}_{\eta}(\boldsymbol{B})\right) \mathrm{d} \eta \\
\boldsymbol{f}(\eta, \boldsymbol{p})=f(\eta,|\boldsymbol{p}|) \frac{\boldsymbol{p}}{|\boldsymbol{p}|} .
\end{gathered}
$$

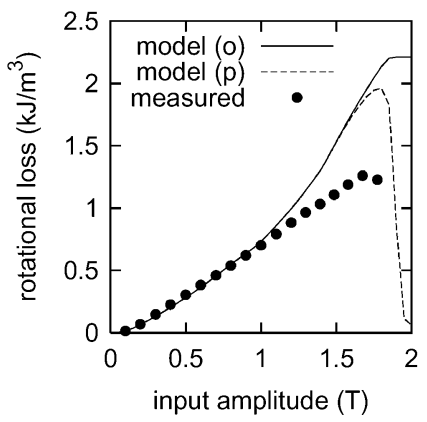

Fig. 4. Rotational hysteresis loss per cycle simulated by the vector play model.

where $f(\eta, p)$ is a shape function. The vector play model having input $\boldsymbol{H}$ has been discussed in [7] and [8].

By setting

$$
f(\eta, p)=f\left(\eta, \pm B_{\mathrm{S}} \mp \eta\right) \text { when } \pm p \geq B_{\mathrm{S}}-\eta
$$

(double signs in same order), the vector play model does not have a hysteretic property when $|\boldsymbol{B}|>B_{\mathrm{S}}$. However, similarly to the stop hysteron, the play hysteron can be redefined as

$$
\boldsymbol{p}_{\eta}(\boldsymbol{B})=\boldsymbol{B}-\frac{\eta\left(\boldsymbol{B}-\boldsymbol{p}_{\eta}^{0 *}\right)}{\max \left(\eta,\left|\boldsymbol{B}-\boldsymbol{p}_{\eta}^{0 *}\right|\right)}
$$

where $p_{\eta}^{0 *}$ is given by (10). This modification does not affect the unidirectional property of the vector play model.

Two types of play hysterons, hysteron (o) - the original type using (1), and hysteron (p) - the modified type using (18) and (10), obviously correspond to the stop hysterons (o) and (p). Accordingly, Table I and Fig. 2 also explain vector relations of play hysterons (o) and (p) for steady-state rotational inputs.

The vector hysteresis model (15) using play hysterons (o) and (p) is referred to as vector play models (o) and (p), respectively, hereafter. The property of vector play model (o) has been discussed in [6].

For an alternating input with amplitude $B_{\mathrm{a}}$, the vector play model (p) yields the same rate-independent hysteresis loss per cycle as the play model (o) when (17) is assumed.

For the rotational input (4), the vector play model (p) with (17) yields a rate-independent hysteresis loss per cycle of

$$
L_{\mathrm{rot}}\left(B_{\mathrm{a}}\right)=-2 \pi \int_{0}^{B_{\mathrm{m}}} f\left(\eta, \sqrt{B_{\mathrm{m}}^{2}-\eta^{2}}\right) B_{\mathrm{a}} \sin \theta_{\mathrm{P}} \mathrm{d} \eta
$$

where $\theta_{\mathrm{P}}$ is the angle between $\boldsymbol{B}$ and $\boldsymbol{p}_{\eta}$ as shown in Fig. 2.

\section{B. Simulation of Vector Property}

The play models (o) and (p) are examined for a non-oriented silicon steel sheet, JIS: 50A1300. The play model is discretized with 76 hysterons and the shape functions are identified using the method described in [4].

Fig. 4 shows the simulated and measured rotational hysteresis losses per cycle. Model (o) yields constant rotational losses for $B_{\mathrm{a}} \geq B_{\mathrm{S}}(=1.9 \mathrm{~T})$. Model (p) gives a rotational hysteresis loss that decreases with an increase in $B_{\mathrm{a}}$ when $B_{\mathrm{a}}$ is large. The discrepancy with the measured loss can be reduced using a weighting function proposed in [6] (see the Appendix).

Fig. 5 compares the amplitude of $\boldsymbol{H}$ and the phase lag of $\boldsymbol{B}$ to $\boldsymbol{H}$ simulated by the stop model (p) and play model (p), where the simulated amplitude of $\boldsymbol{H}$ agrees with the measured one. The play model gives more accurate phase lag than the stop model. 

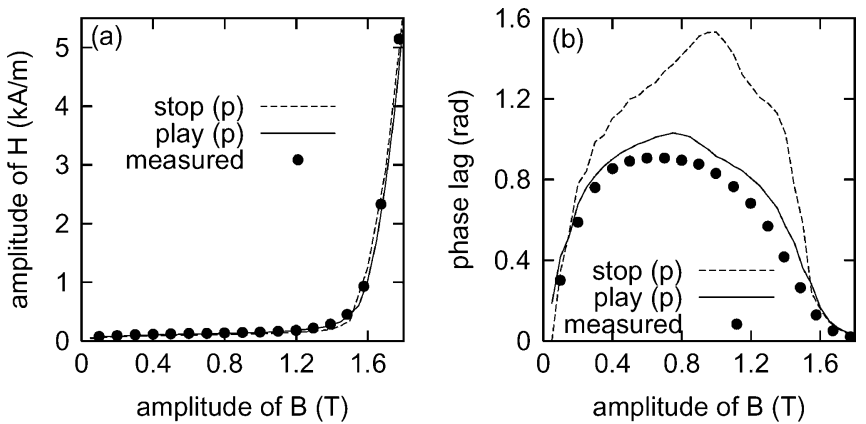

Fig. 5. Amplitude of $\boldsymbol{H}$ and phase lag of $\boldsymbol{B}$ to $\boldsymbol{H}$.
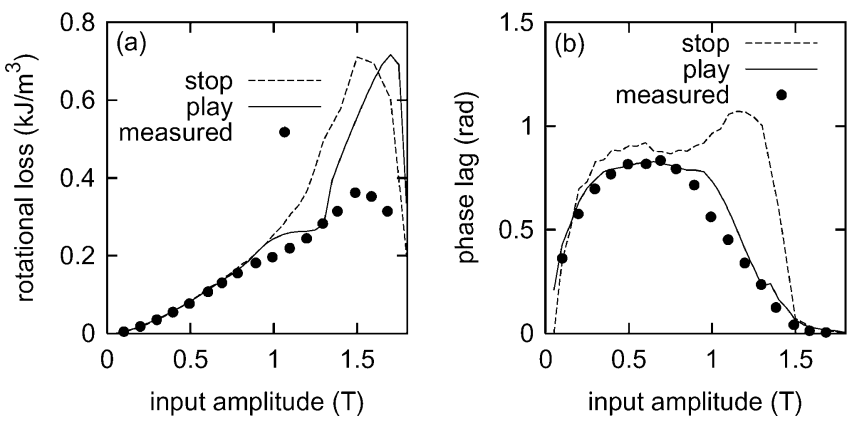

Fig. 6. Simulation results for 35A300. (a) Rotational hysteresis loss. (b) Phase lag of $\boldsymbol{B}$ to $\boldsymbol{H}$.

Simulation results by the stop model (p) and play model (p) for another non-oriented silicon steel sheet (JIS: 35A300) are shown in Fig. 6, which are similar to those for 50A1300. Both models yield large rotational hysteresis losses that can be adjusted to the measured loss as in [6] and [12]. The play model gives more accurate phase lag than the stop model.

\section{CONCLUSION}

Vectorized stop and play hysterons are modified to improve their saturation properties. The stop and play models using the modified hysterons yield magnetic field vectors that are parallel to magnetic flux density vectors under a saturated condition. Comparison with a measured property of the silicon steel sheet shows the modified models obtain rotational hysteretic properties that qualitatively agree with the measured property of the silicon steel sheet.

\section{APPENDIX \\ Loss AdJUSTMENT USING WEIGHTING FUNCTION}

The vector play model having a weighting function is given as

$$
\boldsymbol{P}(\boldsymbol{B})=w(|\boldsymbol{B}|) \int_{0}^{B_{\mathrm{S}}} \boldsymbol{f}\left(\eta, \boldsymbol{p}_{\eta}(\boldsymbol{B})\right) \mathrm{d} \eta
$$

where $w(B)$ is the weighting function. The simulated rotational hysteresis loss can be adjusted to a measured loss by modifying $w(B)$ iteratively [6]. Fig. 7 shows the rotational loss adjusted by several iterations, which coincides with the measured loss of 50A1300. The alternating loss is shown in Fig. 7(b), which is not affected by the modification of $w(B)$ because the shape
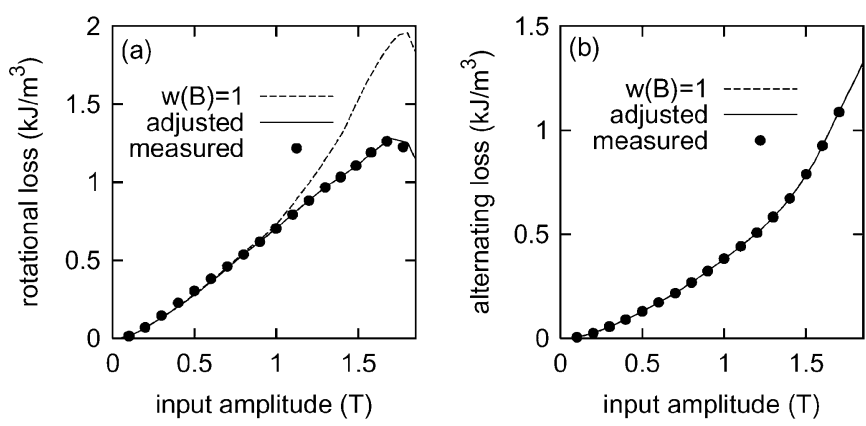

Fig. 7. Hysteresis losses simulated by the play model. (a) Adjusted rotational loss. (b) Alternating loss.

function is identified to reconstruct unidirectional $\mathrm{BH}$ loops corresponding to the modification. The phase lag of $\boldsymbol{B}$ to $\boldsymbol{H}$ is not much affected by the modification. The adjusted rotational loss for $35 \mathrm{~A} 300$ is shown in [6].

The loss adjustment for the stop model is shown in [12].

\section{ACKNOWLEDGMENT}

This work was supported in part by the Japan Society for the Promotion of Science, Grant-in-Aid for Scientific Research (C), 19560288.

\section{REFERENCES}

[1] M. A. Krasnosel'skii and A. V. Pokrovskii, Systems with Hysteresis. Berlin, Germany: Springer-Verlag, 1989.

[2] S. Bobbio, G. Miano, C. Serpico, and C. Visone, "Models of magnetic hysteresis based on play and stop hysterons," IEEE Trans. Magn., vol. 33, pp. 4417-4426, Nov. 1997.

[3] T. Matsuo, Y. Terada, and M. Shimasaki, "Stop model with input-dependent shape function and its identification methods," IEEE Trans. Magn., vol. 40, pp. 1776-1783, Jul. 2004.

[4] T. Matsuo and M. Shimasaki, "An identification method of play model with input-dependent shape function," IEEE Trans. Magn., vol. 41, pp. 3112-3114, Oct. 2005.

[5] T. Matsuo and M. Shimasaki, "A method for optimal identification of a stop model with input-dependent shape function," IEEE Trans. Magn., vol. 42, pp. 3818-3824, Dec. 2006.

[6] T. Matsuo and M. Shimasaki, "Two types of isotropic vector play models and their rotational hysteresis losses," IEEE Trans. Magn., vol. 44, pp. 898-901, Jun. 2008.

[7] A. Bergqvist, "Magnetic vector hysteresis model with dry friction-like pinning," Phys. B, vol. 233, pp. 342-347, 1997.

[8] C. Serpico, M. d'Aquino, C. Visone, and D. Davino, "A new class of Preisach-type isotropic vector model of hysteresis," Phys. B, vol. 343, pp. 117-120, 2004.

[9] J. V. Leite, N. Sadowski, P. Kuo-Peng, and J. P. A. Bastos, "A new anisotropic vector hysteresis model based on stop hysterons," IEEE Trans. Magn., vol. 41, pp. 1500-1503, May 2005.

[10] T. Matsuo and M. Shimasaki, "Isotropic vector hysteresis represented by superposition of stop hysteron models," IEEE Trans. Magn., vol. 37, pp. 3357-3360, Sep. 2001, and its correction, vol. 37, p. 3995, Nov. 2001.

[11] T. Matsuo and M. Shimasaki, "Generalization of an isotropic vector hysteresis model represented by the superposition of stop models-Identification and rotational hysteresis loss," IEEE Trans. Magn., vol. 43, pp. 1389-1392, Apr. 2007.

[12] T. Matsuo, "Comparison of rotational hysteretic properties of isotropic vector stop models," in Proc. 13th Biennial IEEE Conf. Electromagnetic Field Computation (CEFC), Athens, Greece, PB3-15, p. 132, May 2008.

Manuscript received March 03, 2008. Current version published December 17, 2008. Corresponding author: T. Matsuo (e-mail: tmastuo@kuee.kyoto-u.ac. jp). 